\title{
Prêtres de la génération Jean-Paul II : recomposition de l'idéal sacerdotal et accomplissement de soi
}

\section{Céline Béraud}

\section{CpenEdition}

Journals

Édition électronique

URL : http://journals.openedition.org/assr/3345

DOI : 10.4000/assr.3345

ISSN : $1777-5825$

Éditeur

Éditions de l'EHESS

Édition imprimée

Date de publication : 1 mars 2006

Pagination : 45-66

ISBN : 2-7132-2046-7

ISSN : 0335-5985

Référence électronique

Céline Béraud, «Prêtres de la génération Jean-Paul II : recomposition de l'idéal sacerdotal et accomplissement de soi », Archives de sciences sociales des religions [En ligne], 133 । janvier - mars 2006, mis en ligne le 03 mai 2009, consulté le 10 décembre 2020. URL : http:// journals.openedition.org/assr/3345; DOI : https://doi.org/10.4000/assr.3345 


\section{Cêne Béraud}

\section{Prêtres de la génération Jean-Paul II : recomposition de l'idéal sacerdotal et accomplissement de soi}

L'expression « jeune prêtre » dans cette institution dont les clercs présentent une pyramide des âges à la forme des plus singulières, est utilisée de manière fort extensive. Ainsi l'association des "jeunes prêtres " du diocèse de Valence qui organise notamment des journées récréatives, regroupe des personnes nées après 1950. Le présent corpus est constitué de jeunes prêtres ordonnés il y a moins de 10 ans et âgés au plus de 40 ans ${ }^{1}$.

Parler d'une génération Jean-Paul II à propos des jeunes prêtres a du sens pour plusieurs raisons. Il s'agit d'individus, nés après le Concile de Vatican II, dont la socialisation religieuse s'est en grande partie réalisée sous le pontificat du pape polonais. De plus, leur conception de la prêtrise se trouve en nette congruence avec les textes romains parus au cours de la précédente décennie, documents qui revalorisent l'idéal sacerdotal. Enfin, leur papisme prend largement sa source dans l'exemplarité de Jean-Paul II en tant que pleine réalisation de cet idéal. Toutes ces caractéristiques contribuent indéniablement à distinguer ces jeunes prêtres de leurs aînés.

La figure du jeune prêtre est particulièrement énigmatique. « On est une question vivante ", disait l'un d'eux. La marginalité statistique de la vocation presbytérale qui s'inscrit dans un contexte de grande difficulté de transmission des valeurs et

1. Cet article s'appuie sur un travail de terrain dans le diocèse de Paris et celui de Valence : quatorze entretiens ont été menés avec des « jeunes prêtres ». Ce travail de terrain s'inscrit dans un ensemble plus vaste d'une soixantaine d'entretiens réalisés entre février 2001 et juillet 2004 avec des personnes issues de différentes catégories de permanents de l'institution ecclésiale (évêques, théologiens, prêtres, diacres, permanents laïcs), entretiens au cours desquels la variable générationnelle a été très souvent évoquée et discutée par mes interlocuteurs. S'y ajoute l'observation d'une quarantaine d'offices religieux. Voir Céline BÉRAUD, « Nouvelles formes de division du travail religieux dans le catholicisme français. Entre idéal sacerdotal, processus de professionnalisation et accomplissement de soi ", Paris, Thèse de l'EHESS, 2004. Concernant le diocèse de Paris, l'approche qualitative ici développée se trouve en nette congruence avec les résultats de l'enquête quantitative suivante : Louis-Paul AsTRAUD, Les jeunes prêtres : génération Lustiger, Paris, Éditions Golias, 2003. 
pratiques religieuses aux jeunes générations ${ }^{2}$, en est une première raison. Plus radicalement, les stéréotypes qui sont attachés à ces prêtres (crispation identitaire, valorisation du modèle sacerdotal et de sa dimension sacrificielle, tendance à l'autoritarisme $)^{3}$ semblent les placer dans une situation de profonde contradiction avec la modernité. C'est cette hypothèse que nous nous proposons de discuter ici.

\section{Une génération en rupture paradoxale avec celle des années 1960-1970}

\section{Une certaine réactivation de l'idéal sacerdotal}

Les années 1960 et 1970 ont été marquées, dans l'Église catholique, par une profonde mise en question, par les prêtres, de l'idéal sacerdotal qui, depuis plusieurs siècles, fonctionnait comme un modèle, c'est-à-dire un "système symbolique qui dicte la conduite des hommes et la justifie à leurs propres yeux » ${ }^{4}$.

L'idéal sacerdotal est le fruit d'une construction historique et sociale de très long terme qui s'est réalisée en plusieurs étapes. Ainsi la Contre-Réforme, qui constitue indéniablement un moment majeur de ce processus, s'inscrit dans " une culture ancienne de la perfection sacerdotale ${ }^{5}$ : Comme le montre Nicole Lemaitre, "Une idéologie radicale du sacerdoce mis à part s'instaure, dès avant le concile de Trente ${ }^{6}$. Ce moment historique ne constitue donc pas tant une rupture que l'accomplissement d'un certain nombre de quêtes antérieures demeurées jusque-là mal satisfaites. Dès le $\mathrm{IV}^{\mathrm{e}}$ siècle, les principaux traits du modèle sacerdotal commencent à se dessiner : appel à la chasteté, invitation à « mépriser les réjouissances mondaines et les richesses matérielles " ${ }^{7}$ et à se tenir à l'écart, si possible, des formes de travail profanes. L'Église carolingienne place, elle aussi, " très haut l'idéal du prêtre de paroisse ». Elle appelle ce dernier à devenir, grâce à un mode de vie singulier et séparé, un guide exemplaire pour la conduite des fidèles. La réforme du pape Grégoire VII, qui impose le célibat aux prêtres de l'Église d'Occident, prolonge ce mouvement. Cependant, à la fin du Moyen Âge, les écarts entre l'idéal de perfection de vie chrétienne auquel doit tendre le prêtre

2. Yves Lambert, « Les jeunes et le christianisme : le grand défi », Le débat, mai-août 1993, $\mathrm{n}^{\circ} 75$, p. 63-80.

3. On peut notamment se référer aux critiques plutôt sévères de Bernard SEsBouË, N'ayez pas peur! Regards sur l'Église et les ministères aujourd'hui, Paris, Desclée de Brouwer, 1996, p. 31 ; Claude FliPO, "Les jeunes prêtres en France ", Études, nov. 1994, p. 513-523 ; Pierre Pierrard, Un siècle de l'Église de France 1900-2000, Paris, Desclée de Brouwer, 2000, p. 222223.

4. Ce sens du mot "modèle " est celui de Georges Duby tel qu'il est repris par Claude Dubar et Pierre TripIER dans Sociologie des professions, Paris, U. Colin, 1998, p. 15.

5. Nicole Lemaitre, dir., Histoire des curés, Paris, Fayard, 2002, p. 159.

6. Ibid., p. 168.

7. Catherine VinCENT in Nicole Lemaitre, dir., op. cit., p. 25. 
et la réalité, sont encore très grands et constituent un important moteur aux volontés de réformes: "(...) le thème de l'honestas clericorum fait consensus d'un bout à l'autre de l'Europe vers 1480. Il s'agit de lutter contre des "abus" jugés désormais insupportables, mais aussi de réclamer plus de piété, plus d'ascèse, plus de sens du sacrifice de la part de ceux qui sont assimilés au Bon Pasteur par l'idéologie ambiante ${ }^{8}$. Le concile de Trente, véritable exaltation du clergé et du sacerdoce, réaffirme dans ses canons la nécessité du caractère irréprochable de la conduite du prêtre, en lien étroit avec le rôle de médiateur qui lui est assigné. Pour atteindre cet objectif, une forte prise de distance par rapport au monde est plus que jamais préconisée. Ainsi le prêtre doit-il " porter l'habit long et les cheveux courts, ne pas jouer en public ni fréquenter les tavernes, se tenir éloigné des femmes, étudier et prier ${ }^{9}$. Il doit faire preuve de dévouement, d'oubli de soi pouvant aller jusqu'à l'abnégation, voire la dépossession. Ainsi au sein de l'École française de spiritualité, certains courants développent, au XVII siècle, des positions aux nettes dimensions sacrificielles: «(...) l'attention se polarise sur les pratiques ascétiques et plus largement, sur l'anéantissement de la personnalité et l'étroit contrôle des sens et du corps " ${ }^{10}$. Les séminaires, qui se mettent alors progressivement en place, décrits par Bernard Dompnier comme tendant à se réduire "à une sorte de noviciat religieux centré sur la conformation à un règlement tatillon " ${ }^{11}$, sont mis au service de cet objectif de formation de prêtres vertueux et détachés du monde. Les écarts entre ce modèle exigeant et la réalité se réduisent très lentement, même si les comportements déviants demeurent nombreux. Ainsi le "bon prêtre » du XVIII" siècle se définit-il surtout en creux comme quelqu'un qui «fuit les excès » et "mène une existence séparée ${ }^{12}$. Il ne faut pas pour autant sous-estimer la force de l'idéal sacerdotal renforcé par le concile de Trente et l'École française, mis en application par les sociétés de prêtres séculiers telles que les sulpiciens, les lazaristes et les oratoriens, puisque celui-ci continue à façonner le modèle clérical pendant plusieurs siècles. Ainsi, selon Pierre Pierrard, la spiritualité du prêtre demeure-t-elle "inchangée » ${ }^{13}$ au début du XIX ${ }^{\mathrm{e}}$ siècle. L'historien renouvelle ce constat un siècle plus tard: "Entre les deux guerres, la formation des futurs prêtres dans les séminaires français (...) n'a guère changé depuis deux siècles. Les manuels, règlements et directoires viennent en droite ligne du XVII ${ }^{\mathrm{e}}$ ou du XVII ${ }^{\mathrm{e}}$ siècle ${ }^{14}$. Les prêtres qui ont vécu Vatican II avaient

8. Nicole Lemaitre, op. cit., p. 168.

9. Viviane BARRIE-CURIEN, Marc VénARD, «Les clergés " in Marc VéNARD, dir., Histoire du christianisme. Tome 8, le temps des confessions (1530-1620), Paris, Desclée, 1992, p. 913.

10. Bernard DOMPNIER, "Continuité de la réforme catholique " in Marc VÉNARD, dir., Histoire du christianisme. Tome 9, l'âge de raison (1620/30- 1750), Paris, Desclée, 1997, p. 271.

11. Ibid., p. 271.

12. Ibid., p. 278.

13. Pierre Pierrard, Le prêtre français du Concile de Trente à nos jours, Paris, Desclée, Bibliothèque d'Histoire du Christianisme, Paris, 1986, p. 84.

14. Ibid., p. 123. 
été socialisés dans ce type d'institutions ${ }^{15}$. Ils se trouvent imprégnés du modèle sacerdotal qu'un certain nombre vont rejeter.

Dès l'entre deux guerres, cette construction idéologique extrêmement puissante entre en crise. La contestation cléricale qui atteint son apogée à la fin des années 1960 avec un groupe comme "Échanges et Dialogue ", vise une "désacerdotalisation" de la prêtrise. Elle s'organise autour de quatre axes principaux : l'accès au travail salarié, à l'engagement politique et syndical, au mariage, à la participation de type démocratique aux décisions de l'Église ${ }^{16}$. Symboliquement, c'est l'obligation du port de la soutane qui est abandonnée dès 1962 dans le diocèse de Paris. Ces revendications participent toutes de la même volonté de «se fondre dans la masse ${ }^{17}$ et d'en finir avec le traitement jugé quelque peu infantilisant que leur assure l'Église. Dans une perspective d'évangélisation de milieux sociaux de plus en plus éloignés de la foi, il s'agit de "sortir du presbytère " ${ }^{18}, \mathrm{~d}$ ' «aller au monde » et de s'y intégrer jusqu'à l'enfouissement. Ce projet est développé dès les années 1930 par les aumôniers des mouvements d'Action Catholique, puis pendant la guerre par les prêtres qui font l'expérience du STO. L'atteinte portée à l'intégrité sacerdotale par l'incarnation mondaine que la vie en milieu ouvrier exige, au moins autant que les liens tissés sur le terrain avec le marxisme en plein contexte de guerre froide, amène Pie XII à condamner l'expérience des prêtres ouvriers. Selon Martine Sévegrand, la crise qui éclate au grand jour, dans les années 1960, est étroitement liée à cette controverse récurrente depuis l'entre-deux-guerres : «La "spiritualité" qui se cherche veut conjuguer sacerdoce et incarnation, vie liturgique et action, prière et évangélisation ; elle dessine un prêtre diocésain qui s'éloigne du statut du religieux pour retrouver les hommes dans le concret de leur existence ${ }^{19}$. Le concile, qui suscite un immense espoir chez les partisans du changement, conduit certes à une conception rééquilibrée du rôle du prêtre, entre évangélisation et prise en charge des fonctions liturgiques. De plus, dès 1966, s'engage une réorganisation en profondeur des séminaires. Cependant, les déceptions qui se font alors jour, sont liées à l'absence de renouvellement en profondeur de la spiritualité du prêtre ainsi qu'au refus manifesté par l'institution ecclésiale de diversifier les voies d'accès à ce ministère. On retrouve ici deux conceptions du prêtre qui historiquement, au nom d'une interprétation différente de la tradition, se sont affrontées dans le catholicisme : celle de l'homme de l'autel et du sacré d'une part, celle du ministre

15. Charles SuAud, La vocation. Conversion et reconversion des prêtres ruraux, Paris, Éditions de Minuit, 1978.

16. Denis Pelletier, La crise catholique. Religion, société, politique, Paris, Payot, 2001, p. 62 ss.

17. Luc Perrin in Nicole Lemaitre, dir., op. cit., p. 403.

18. Expression employée par Pierre PIERRARD (1986), op. cit., p. 103, à propos du tout début du Xx $\mathrm{Xx}^{\mathrm{e}}$ siècle et qui convient aussi très bien aux années 1960.

19. Martine Ṡ̀VEGRAND, Vers une Église sans prêtres. La crise du clergé séculier en France (1945-1978), Rennes, PUR, 2004, p. 33. 
de l'Évangile d'autre part ${ }^{20}$. Les mouvements contestataires, sur fond d'assèchement des vocations et de vagues de départs massifs, reflètent aussi l'aspiration des prêtres à accéder aux valeurs individualistes et hédonistes propres à la modernité qui pénètrent alors le champ religieux. "Ce qui est en cause, c'est (...) le renoncement à l'idée du sacrifice de soi et la réévaluation de l'épanouissement du prêtre dans sa condition de prêtre ${ }^{21}$, résume Denis Pelletier. Dès lors, le modèle sacerdotal semble socialement disqualifié. Non seulement, comme l'écrit Danièle Hervieu-Léger, « (...) la vision du prêtre-hostie, mortifiant sa chair pour la plus grande gloire de Dieu, est devenue socialement et psychologiquement irrecevable ${ }^{22}$, mais elle est aussi devenue contre-productive d'un point de vue pastoral. Dans cette perspective, la « crise des vocations » a même pu apparaître a posteriori comme "la première grande vague [du] processus d'émancipation religieuse ${ }^{23}$ alors en cours.

Cet idéal brocardé dans les années 1960 et 1970 se trouve aujourd'hui réactivé. On touche ici à un premier paradoxe : cette réactivation est-elle un pur archaïsme ?

\section{Une resacerdotalisation qui se veut heureuse}

La seconde différence majeure avec la période actuelle réside dans la thématique du prêtre comme homme en souffrance du fait de la crise de son statut social, très en vogue pendant les années de la contestation. Dans une société sécularisée et urbanisée, le prêtre ne fait plus partie des notables. Il se trouve en marge des grandes institutions intégratrices que sont le travail et le couple. Il ne peut plus avoir prétention à régenter idéologiquement les fidèles dans leur vie quotidienne. Enfin, son monopole au sein de l'institution ecclésiale se trouve mis en question par les diacres permanents dont le ministère a été réhabilité par Vatican II ainsi que par les laïcs auxquels le concile a prêté une attention particulière. Dans son ouvrage autobiographique, François Charles insiste sur l'incompatibilité même qui apparaissait aux yeux des hommes de sa génération entre la prêtrise et le bonheur : «Le fait de savoir si un prêtre était heureux ou non ne nous intéressait pas. Plus! Nous aurions été quelque peu scandalisés d'apprendre qu'un prêtre pouvait se soucier d'être heureux en ce monde. Si nous avions

20. Voir, comme exemple de discours contestataire (quoique pas radical) ancré dans une lecture de l'histoire, Daniel OlIVIER, Les deux visages du prêtre. Les chances d'une crise, Paris, Fayard, 1971. Significatif d'un même type de réflexion, mais moins contestataire et antérieur, voir aussi Jean Colson, Ministre de Jésus-Christ ou le sacerdoce de l'Évangile. Études sur la condition sacerdotale des ministres chrétiens dans l'Église primitive, Paris, Beauchesne, 1966.

21. Denis Pelletier, op. cit., p. 68.

22. Danièle Hervieu-LéGer, Postface à l'ouvrage de François Charles, La Génération défroquée, Paris, Cerf, 1986, p. 142.

23. Yves LAMBert, Dieu change en Bretagne. La religion à Limerzel de 1900 à nos jours, Paris, Cerf, 1985, p. 295. 
cherché à être heureux, nous ne nous serions pas fait prêtres (...) On ne peut pas à la fois racheter le monde par la croix et être heureux ${ }^{24}$.

Depuis le début des années 1990, les ouvrages témoignages émanant d'évêques et de jeunes clercs constituent une abondante littérature. Ils participent d'un véritable marketing du bonheur. Parmi les nombreux récits que nous pourrions citer $^{25}$, celui de Patrice Gourrier est très représentatif de cette nouvelle rhétorique : «(...) je suis toujours peiné d'entendre: "Je vous admire, mon Père car vous avez sacrifié votre vie." Je n'ai nullement l'impression d'avoir sacrifié ma vie, bien au contraire! Suivre le Christ, au milieu du monde où je vis, me rend parfaitement heureux, même si cela ne m'épargne pas les difficultés de l'existence. Présenter la vie du prêtre comme celle d'un "sacrifié" n'est ni encourageant ni motivant ${ }^{26}$. Il est particulièrement intéressant de noter que dans l'enquête réalisée par Christian Baudelot et Michel Gollac sur "travail et bonheur ", le groupe qui exprime le plus haut degré de satisfaction professionnelle est le clergé $(80 \%$ des personnes interrogées), suivi de près par les artistes et journalistes $(78 \%)$, mais loin devant les professeurs (65\%), les travailleurs sociaux (60\%) et surtout les militaires et policiers $(46 \%)^{27}$. Au cours des entretiens menés avec des jeunes prêtres, ressort généralement la volonté d'afficher qu'ils sont des hommes épanouis, équilibrés et heureux, avec une assurance pouvant friser chez certains l'arrogance. Ils semblent sortis, dans leur majorité, de la thématique des renoncements douloureux au profit d'un discours fondé sur la liberté.

«Pour moi, engagement définitif et bonheur sont compatibles, contrairement à ce que pensent certains. Sinon, je ne serais pas là. Je n'ai pas une spiritualité masochiste, ni sacrificielle!». Prêtre du diocèse de Valence, âgé de 35 ans, ordonné deux ans auparavant.

«Je n'ai pas choisi la prêtrise par mortification, par goût du sacrifice. Je pressentais qu'il y avait un bonheur possible dans cette vie-là ». Prêtre du diocèse de Valence, âgé de 29 ans, ordonné deux ans auparavant.

D'ailleurs beaucoup évoquent la rencontre avec des prêtres rayonnants comme l'un des éléments décisifs de leur vocation. Dans un document récent, les

24. François CHARLES, op. cit., p. 13. L'auteur fait partie des 1500 prêtres environ qui ont quitté le sacerdoce entre la fin des années 1960 et le début des années 1980.

25. La thématique du bonheur telle un passage obligé, est présente dans la plupart des ouvrages traitant de la prêtrise parus au cours de la décennie passée. Voir par exemple, Michel Mounier, Bernard Tordi, Les prêtres... tout simplement, Paris, Éditions de l'Atelier, 1994, p. 49 ; 212-213 «Le célibat : épanouissement et témoignage »; Bernard SESBOUË, op. cit., p. 66 ; Claude Goure, Cinq prêtres dans l'histoire. Confidences de 1944 à nos jours, Paris, Bayard, 2004, p. 49 "Heureux dans ce "métier"-là »; Hippolyte SIMON, Libres d'être prêtres, Paris, Éditions de l'Atelier, 2001 (cf. chap. IV).

26. Patrice Gourrier, J'ai choisi d'être prêtre. Un autre regard sur le monde. Entretien avec Jacques Rigaud, Paris, Flammarion-Desclée de Brouwer, 2003, p. 111.

27. Source: "Enquête statistique sur la satisfaction au travail en France (1996-1999) », Laboratoire de l'École normale supérieure de la rue d'Ulm-INSEE. Résultats publiés et commentés dans Christian BAUdelot, dir. et Michel GollaC, dir., Travailler pour être heureux? Le bonheur et le travail en France, Paris, Fayard, 2003. 
évêques de France "proclament leur intense désir que des baptisés (...) accèdent librement, courageusement et avec bonheur à l'ordination ${ }^{28}$, en insistant longuement sur «la joie de servir comme prêtre ${ }^{29}$. Dans cette même perspective, le Cardinal Lustiger affirme : "La fidélité aux conseils évangéliques (puisque c'est ainsi que la Tradition a nommé l'engagement aux renoncements de la pauvreté, de l'obéissance et de la chasteté) n'est donc pas une abnégation suicidaire, mais partage et imitation de l'amour de Jésus. C'est une grâce dont la gratuité comble et éblouit. Voilà pourquoi elle peut être librement vécue dans la paix et dans la joie ${ }^{30}$.

Comment expliquer la réactivation de l'idéal sacerdotal alors que ses dimensions sacrificielles semblent être en profonde contradiction avec les exigences de la modernité religieuse ? Comment la même génération est-elle parvenue à accéder à une réalisation de soi affichée comme heureuse dans la vocation presbytérale tout en réactivant un idéal dont les dimensions sacrificielles avaient tant fait souffrir leurs aînés ? Dans l'ouvrage qu'il consacre aux religieuses, Julien Potel, après avoir rappelé les vœux d'obéissance, de pauvreté et de chasteté, prononcés par ces dernières, se trouve confronté au même type d'énigme: "Vouloir se réaliser soi-même et obéir en religieuses (...) Dans notre société où l'on veut développer toutes ses possibilités et se dépasser, alors que l'initiative et la liberté de chacun sont capitales, quelle est la signification de l'obéissance des religieuses auprès des femmes et des hommes d'aujourd'hui " ${ }^{31}$. À cette situation paradoxale, le sociologue ne donne pas de réponse et laisse la place au prêtre qu'est aussi Julien Potel, en évoquant la signification spirituelle de ces renoncements.

\section{Une figure du prêtre aux allures néo-tridentines}

Attachement à des attributs distinctifs (recherche d'une forte visibilité sociale) ainsi qu'aux dimensions sacrificielles de l'idéal sacerdotal qui font du prêtre un homme séparé, exemplaire, et autoritarisme, sont les principaux éléments du modèle sacerdotal qui sont aujourd'hui réactivés et qui donnent aux jeunes clercs des allures néo-tridentines.

\section{Distinction et idéal sacerdotal}

Les jeunes prêtres ont tous une profonde conscience de la singularité de leur choix de vie ainsi que de la perception sociale de celle-ci, comme l'illustrent les témoignages suivants :

28. LeS ÉVÊQUeS DE FranCE, La formation des futurs prêtres, Paris, Le Centurion-Cerf, 1998 , p. II.

29. Ibid., p. 15 et ss. p. 109.

30. Jean-Marie Lustiger, Les prêtres que Dieu donne, Paris, Desclée de Brouwer, 2000,

31. Julien Potel, Portes ouvertes chez les religieuses. Propos d'un sociologue. Échos des médias, Paris, L'Harmattan, 1999, p. 310. 
«Il y a quelque chose de fou dans ce type d'engagement. Ce n'est pas neutre comme témoignage. Ça dérange dans un sens ou dans l'autre ». Prêtre du diocèse de Paris, 31 ans, ordonné un an auparavant.

"Je pense que certaines personnes doivent associer notre vocation à une espèce de folie. Folie, au sens où l'on s'astreint à des choses. Ça, ça peut entraîner du respect ou du dénigrement ". Prêtre du diocèse de Paris, 36 ans, ordonné 8 ans auparavant.

"Ce qui me saute aux yeux, c'est l'aspect marginal de notre situation. Je ressens très fortement la marginalité de nos choix (...) Je pense qu'il y a des gens qui nous voient un peu, pas comme des martyrs, mais des personnes qui ont pris un engagement dont ils ne se sentent pas la force. Il y a une sorte d'héroïsme qui fait que la figure du prêtre reste loin... Il y a aussi de l'indifférence ou de la curiosité. Les gens ne savent pas grand-chose de la vie des prêtres. C'est vrai que le domaine affectif arrive très vite dans les conversations. Les conditions de vie aussi, l'aspect financier des choses ». Prêtre du diocèse de Valence, 31 ans, ordonné il y a 4 ans.

La plupart des jeunes prêtres ont recours spontanément, au cours de l'entretien, au terme " radicalité » pour décrire leur vocation. C’est, en effet, dans la rupture avec certaines normes et valeurs dominantes comme la recherche du plaisir et celle du profit, que se situe leur particularité. L'idéal sacerdotal leur offre, dans cette perspective radicale, un ensemble très précieux de ressources mobilisables.

Les pratiques distinctives les plus évidentes résident dans les attributs vestimentaires. De façon plus subtile, l'attachement scrupuleusement affiché au respect de la normativité canonique y participe aussi pleinement, le célibat constituant à ce niveau la meilleure illustration. À un moment où la vocation ministérielle est devenue statistiquement rarissime, celle-ci s'impose forcément comme un choix individuel assumé et non le fruit d'une pression sociale. Elle prend, dès lors, une nette valeur distinctive. Port d'un signe extérieur de reconnaissance et respect de la norme peuvent se renforcer l'un l'autre. Un prêtre de Paris me faisait part de l'utilité du col romain, même s'il semblait conscient des limites de sa démonstration :

«Je reprendrais toujours Jean 17. Un vieux prêtre m'avait dit ça en parlant de la soutane : c'est un moyen de se protéger contre les autres et de se protéger contre soimême. Quand j'ai mon col romain... Pardonnez-moi cette image... Mais je ne pourrais pas rentrer dans un sex-shop avec mon col romain. Je me protège. J'ai le respect de l'uniforme... Le col romain, c'est un degré d'exigence. Je ne fais pas ce que je pourrais faire... Bon en même temps, ce n'est pas l'habit qui fait le moine. Ce que j'ai fait, je l'ai fait aussi parce que c'est en moi. Disons que ça prévient, dans tous les sens du terme ". Prêtre âgé de 36 ans, ordonné depuis 6 ans.

À propos du port de la soutane, le même type d'argumentation était développé au XIX ${ }^{e}$ siècle. On ne peut qu'être surpris par la très grande proximité entre les propos de notre jeune prêtre parisien et ceux qui suivent de Monseigneur Devie, ancien directeur de séminaire, qui écrivait en 1842 : «L'habit ecclésiastique inspire je ne sais quel sentiment de respect religieux qui est aussi utile à celui qui le porte qu'à ceux qui l'entourent (...) Un ecclésiastique en soutane serait remarqué et blâmé s'il entrait au cabaret, dans une salle de spectacle, dans une maison suspecte (...) Tout le monde serait étonné et indigné, si, dans une maison 
honnête, un ecclésiastique, revêtu de son habit, se permettait des conversations légères, des paroles à double sens, des manières peu convenables : tant il est vrai que le costume devient un préservatif pour celui qui le porte ${ }^{32}$. Par l'austérité et le refus des modes vestimentaires qu'il manifeste, le port du col romain accompagné d'habits de couleurs sombres, participe de l'ascèse sacerdotale. Il est l'une des manifestations les plus apparentes (car exposée aux yeux de tous et pas seulement au cours de la liturgie au cercle restreint des pratiquants réguliers) de la radicalité de ce type de vocation. Il contribue à marquer une distance sociale entre le prêtre et le monde dans lequel il évolue. Cette attention particulière portée à l'apparence participe de la "dialectique du regard » développée au XVII siècle par Tronson dans ses Examens particuliers, dialectique qui gagne fortement en acuité dans le contexte actuel où les clercs sont rares et méconnus : «(...) si le prêtre ne doit pas avoir les yeux fixés sur le monde où il risque de se perdre, il ne doit en revanche jamais oublier qu'il est observé par lui. D'où cette insistance majeure sur la composition du personnage extérieur : l'apparence est ici voie d'accès à l'essence du Christ-prêtre dont tout le comportement sacerdotal doit en quelque sorte offrir une transcription lisible à ses contemporains ${ }^{33}$. L'habit et plus largement l'hexis corporelle qui, dans le contexte de l'École française de spiritualité, s'inscrivaient dans les registres de la prescription et du conformisme produits par l'appartenance au corps sacerdotal, sont aujourd'hui les signes d'une identité singulière pleinement choisie par les individus. Comme l'affirme le politiste Philippe Portier à propos des " catholiques de l'identité " dont sont originaires aujourd'hui un grand nombre des jeunes prêtres, "stratégies d'affirmation de foi et d'affirmation de soi » vont désormais de pair ${ }^{34}$. De plus, alors que le port imposé de la soutane avait pour objectif de faire disparaître l'individu derrière sa fonction et son corps d'appartenance, porter librement le col romain est un moyen de faire apparaître socialement une institution aujourd'hui largement invisible. Ainsi alors que le clerc se trouvait entièrement défini par l'institution ecclésiale devant laquelle il se devait de s'effacer, cette dernière n'existe aujourd'hui qu'à travers l'action volontaire d'individus pleinement autonomes. En 1990 encore, Louis Trichet pouvait écrire : «Dans notre pays, la publication du code de 1983 n'a rien changé à la pratique. Le port de la soutane devient de plus en plus exceptionnel en dehors des milieux traditionalistes. Le col romain lui-même a de moins en moins d'adeptes. Beaucoup de prêtres s'habillent comme les hommes de leur milieu (...) $»^{35}$. Notons que si la grande majorité des jeunes prêtres parisiens ont adopté le col romain, c'est le cas d'un seul prêtre du

32. Cité par Philippe Boutry, Prêtres et paroisses au pays du Curé d'Ars, Paris, Cerf, 1986, p. 212.

33. Dominique Julia, "Le prêtre » in L'homme des Lumières, Paris, Seuil, 1996, p. 412-413.

34. Philippe PORTIER, Jean BAUDOIN, dirs., Le mouvement catholique français à l'épreuve de la modernité. Enquête autour d'une militance éclatée, Rennes, Presses Universitaires de Rennes, 2002.

35. Louis TRIChET, La tonsure, Paris, Cerf, 1990, p. 184. 
diocèse de Valence dans lequel cette pratique demeure controversée entre prêtres de générations différentes mais aussi au sein du groupe des jeunes prêtres. Le port de l'habit ecclésiastique y est, en effet, encore fortement associé dans la mémoire collective cléricale à la rigueur disciplinaire d'avant le concile :

« Ici, il n’y a qu'un prêtre qui met le col romain régulièrement. Moi, j’avoue que je ne suis pas assez fort pour rester paisible. On m'enverrait des fions et je répondrais. Heureusement, le salut de l'âme ne se résume pas au clergyman sinon Jésus nous l'aurait dit! Dans notre diocèse, ça renvoie à des choses du passé un peu difficiles. Le grand séminaire de Viviers, c'était plutôt crispé. Il y a des prêtres âgés qui en ont bavé ». Jeune prêtre du diocèse de Valence.

Le retour à des usages quelques peu tombés en désuétude concernant la dénomination des prêtres s'inscrit aussi dans cette même quête de visibilité et de distinction. Ainsi sur les cartes de visite de certains jeunes clercs et, plus inattendu, sur leur adresse électronique, le nom de famille est précédé du titre «Abbé » ou "Monsieur l'Abbé » ${ }^{36}$. Il s'agit là encore d'une tentative de restauration de l'institutionnalité catholique à partir de ses membres.

Selon un processus assez classique de retournement du stigmate, c'est dans leur marginalité même, vécue comme socialement disqualifiante dans les années 1960-1970 et désormais présentée comme rupture prophétique, que les jeunes prêtres trouvent des sources de distinction. Une telle attitude, qui apparaît socialement comme étant «à contre-courant ${ }^{37}$ pour reprendre le titre d'un chapitre de l'ouvrage du Cardinal Lustiger consacré aux jeunes prêtres, renoue avec l'intransigeantisme catholique face au monde moderne. Ainsi, au cours des entretiens, l'évocation de l'attachement à la chasteté ${ }^{38}$ et des interrogations sociales qu'elle suscite, passe-t-elle fréquemment par un dénigrement des mœurs actuelles. «Comment voulez-vous que les gamins comprennent ce qu'est la chasteté vu le comportement de leurs parents... Dans mon ancienne paroisse, plus populaire, combien de fois j'ai eu des gamins qui sont venus sonner chez moi, me disant qu'ils ne pouvaient pas rentrer chez eux parce que leur mère était avec leur mec... ", me disait un jeune prêtre parisien. La distance prise par rapport au pouvoir, à l'argent et à la sexualité, participe d'une stratégie compensatoire pour les clercs dont le statut s'est trouvé tout à la fois banalisé et déclassé. L'idéologie sacrificielle partie prenante du modèle sacerdotal, se trouve ici mobilisée. Elle s'inscrit dans le registre de la performance de l'ascèse qui exerce une certaine

36. Remarquons que ce réemploi est pour le moins surprenant puisque le titre « Monsieur l'Abbé » renvoyait à la possibilité dans le système bénéficial pour les clercs réguliers d'être dotés d'une abbaye. Il n'a donc aucun contenu religieux ou spirituel proprement dit (contrairement à l'expression " Mon Père »). Élément parfaitement obsolète, il ne vise aujourd'hui qu'à réinscrire la prêtrise dans la mémoire reconstruite d'un temps passé et idéalisé.

37. Jean-Marie LusTigER, op. cit., p. 105.

38. Tous les chrétiens sont appelés à la chasteté : dans la continence s'ils sont célibataires, dans la chasteté conjugale s'ils sont mariés. Voir Catéchisme de l'Église catholique, Paris, CenturionFleurus-Mame-Librairie éditrice Vaticane, 1992, $\mathbb{} 2348$ et ss. 
fascination sociale. Plus le renoncement est grand, plus la performance est remarquable. Ainsi dans les biographies dressées chaque année par la revue diocésaine Paris-Notre Dame à l'occasion des ordinations du mois de juin, le cursus scolaire brillant de certains séminaristes (tout particulièrement le passage par une grande école prestigieuse offrant de belles perspectives de carrière) se trouve-t-il mis en valeur.

On remarque que si le nombre de prêtres religieux est décroissant, sa baisse est moins accentuée que celle des séculiers ${ }^{39}$. C'est en grande partie dans le caractère radical de la vocation monastique, en rupture flagrante avec les valeurs intramondaines, que l'on peut trouver une explication à ce phénomène. La fascination sociale pour l'ascèse se trouve davantage satisfaite par la figure du clerc régulier et tout spécialement du moine que par celle du séculier. Le premier, par la performance qu'il réalise sur lui-même, apparaît donc comme l'une des figures les plus nobles du virtuose religieux. Le séculier au contraire voit son sacerdoce en quelque sorte affadi par les compromissions que sa vie dans le monde peut lui faire connaître.

Dans la même perspective, les médias se sont régulièrement fait l'écho admiratif des opinions publiques par rapport au courage de Jean-Paul II (si souvent décrié par ces mêmes médias pour ses positions éthiques) lors des ses déplacements à travers le monde malgré son grand âge et sa santé des plus fragiles ${ }^{40}$. En 1980 déjà, le Cardinal Marty le qualifiait d' "athlète de Dieu ". Cette remarquable performance a fait accéder le pape au rang des héros contemporains.

\section{La nécessaire exemplarité du prêtre}

Dès lors, tout ce qui porte atteinte à l'exemplarité de la figure du prêtre peut se trouver dénoncé, y compris des comportements très répandus et fort banals : "(...) à mon arrivée dans la région, j'étais fumeur : que n'ai-je pas entendu sur ce sujet ! "Comment, vous, un prêtre, vous vous livrez à ces passions sordides ?" J'ai arrêté de fumer, comme d'aller vite en voiture, pour ne pas trop choquer les gens $(. ..){ }^{41}$. Plus sulfureuse, la rupture de l'engagement au célibat, sorte de contre-performance, n'est pas rare. Chaque année, une dizaine de prêtres quittent

39. Ainsi entre 1991 et 2000, l'effectif des prêtres diocésains en passant de 24624 à 19234 connaît une baisse de 21,89\%. Celui des prêtres religieux en passant de 6940 à 6119 , ne diminue que de 11,83\%. Source : Conférence des Évêques de France, 2004.

40. C'est en ayant recours à la thématique de la performance que le journal Libération a rendu compte de la venue du pape à Lourdes pour le 15 août 2004 : "Parfois, la caméra qui transmet les images aux écrans géants installés sur les sanctuaires se détourne de la lippe bavante, de sa main tremblante qui s'accroche à un mouchoir blanc. Jamais un fidèle ne dit que l'apparence du pape est obscène. Mais en l'applaudissant à chaque fois qu'il doit interrompre son discours les pèlerins ont tout l'air de saluer une "performance” ", Jacky DuRAND, envoyé spécial à Lourdes, Libération, lundi 16 août 2004.

41. Père Olivier-Marie, Curé de campagne, Paris, Arléa, 2003, p. 27. 
le sacerdoce, souvent afin de se marier. Ces départs ${ }^{42}$, à mettre en comparaison avec la moyenne annuelle de 120 ordinations, sont d'ailleurs peu ébruités. Ils suscitent cependant, en interne, une certaine agitation au sein des diocèses concernés. Comme le souligne François de Singly, nos contemporains « oscillent entre l'admiration pour les gens qui ne changent pas, pour leur fidélité à leur engagement et le doute qu'il n'y a que les imbéciles qui ne changent pas ${ }^{43}$. En 2002, un prêtre du diocèse de Valence ordonné en 2000 a renoncé à la prêtrise pour épouser une jeune femme qu'il avait rencontrée. La plupart de ses confrères avec lesquels je me suis entretenue à l'époque, ont évoqué ce cas :

«Là, $j$ ’ai un ami qui vient de quitter le ministère. J'en ai discuté avec certaines personnes.

À la fois, on dit: le célibat, c'est nul. Mais la rupture par rapport à cet engagement au célibat, ça casse des choses profondes chez ceux qui ont côtoyé ce prêtre de voir qu'il a quitté le ministère... C'est une occasion d'approfondir sa foi certainement. Mais, en même temps, ça fait du dégât... Le fait qu'il ait rompu son engagement. Il a fait le choix de quelqu'un au lieu des groupes qui lui étaient confiés. Les gens le perçoivent assez intuitivement. Pour les gens dont il avait été le pasteur, ça a été difficile. Comme dans une famille, quand il y a un divorce. Ça fait du remous et c'est difficile à encaisser... Tout ça, ça dit quelque chose de ce que l'on attend du prêtre. Le célibat, c'est le signe qu'on donne sa vie aux autres. Rompre ça, c'est comme une trahison... » Jeune prêtre du diocèse de Valence.

Plus grave, le scandale de la pédophilie qui a profondément secoué les catholiques américains et qui n'épargne pas l'Église de France ${ }^{44}$, se trouve en profonde contradiction avec l'idéal de sainteté du prêtre. Un certain nombre de ceux que j'ai rencontrés, ont évoqué ce thème spontanément. La suspicion généralisée qui en résulte est fort mal vécue. Ainsi un prêtre parisien me citait le cas d'un groupe d'aumônerie de collégiens à qui il a fait rencontrer un séminariste. Les adolescents ont dit au jeune homme : "Tu ne vas pas devenir prêtre, tu vas être pédophile ». Il en résulte l'élaboration de stratégies d'évitement de tout contact individualisé et isolé avec des enfants ou des adolescents.

42. Le nombre de départs est grossièrement évalué, faute de statistiques officielles en la matière. Selon le quotidien La Croix, ce serait dix prêtres qui en moyenne renonceraient à leur ministère chaque année. Le groupe Jonas, suite à une enquête réalisée dans les diocèses en 2000, avance le chiffre de 100 départs au cours des cinq dernières années, soit une moyenne annuelle de 20 personnes (on peut retrouver les résultats et le commentaire de ce document intitulé, "Enquête de Jonas sur de nouveaux départs de prêtres ", sur internet à l'adresse suivante http://www.groupes-jonas.com). Claude Barthe avec 15 défections par an, propose une évaluation médiane (voir "Crise du sacerdoce en France : la solution du P. Moingt ", Catholica, n 77, automne 2002).

43. François de Singly, Les uns avec les autres. Quand l'individualisme crée du lien, Paris, Armand Colin, 2003, p. 70.

44. Il est important de rappeler que l'Église de France n'est proportionnellement pas plus touchée que d'autres institutions (l'Éducation Nationale par exemple) par les affaires de pédophilie et que son épiscopat semble avoir gérer la crise avec plus de dignité qu'aux États-Unis. Il n'en demeure pas moins qu'un fort climat de suspicion s'est instauré dans notre pays. 


\section{Un attachement farouche et parfois autoritaire aux prérogatives qui sont celles du prêre}

La liturgie, qui a pris une place particulière au sein de certaines filières de formation ${ }^{45}$, a acquis, pour ces jeunes prêtres, une dimension spécifique. Ils remettent également au premier plan les sacrements, tout particulièrement le sacrement de réconciliation ${ }^{46}$ à l'occasion de l'accompagnement spirituel de fidèles. On peut y voir une réaction par rapport au mouvement de désacralisation qui a eu lieu dans les années 1960 et 1970 . Notons que ce recentrage sur le cultuel constitue aussi une modalité de recharge de l'autorité du statut clérical puisque les prêtres y détiennent un monopole plus difficilement contestable. Alors que dans certains domaines de leur ministère ils peuvent se trouver (en ce qui concerne les compétences mobilisées) sur un pied de quasi-égalité professionnelle avec des laïss permanents, ils demeurent en position dominante dans le registre de la sacralité.

La mise en avant intransigeante d'une conception radicale de la prêtrise, vécue sur un mode identitaire fort, ne va pas sans poser problème dans les rapports de collaboration que les jeunes prêtres sont amenés à avoir avec les laïcs, les diacres, mais aussi avec leurs confrères plus âgés. Les deux témoignages suivants en offrent une double illustration.

"Lors de la journée de rentrée sur ma paroisse, j’ai rencontré un jeune prêtre qui vient d'arriver. On ne le sent pas très à l'aise avec nous. Pourtant, on s'est présenté. On a parlé. Je sentais qu'il était réticent. Je pense qu'ils ont peur qu'on leur ouvre les yeux sur des problèmes qu'ils ne veulent pas voir. Rencontrer une femme, c'est parler d'enfants, de contraception, de pilule, d'avortement... Là, ils ne veulent pas entendre. Le Pape a dit, donc... ». Assistante laïque en pastorale du diocèse de Paris.

«Moi j'ai des collaborations avec des gens plus âgés, différents, d'autres milieux... qui peuvent être merveilleuses. D'autres qui sont insupportables parce que le rapport n'est pas juste. Il peut y avoir parfois une espèce de méfiance, des jeux de pressions. La paroisse comme un lieu qu'il faut gérer, dont on veut optimiser la gestion, alors que pour moi, c'est plus un lieu de mission... Dès lors, ça devient un enjeu de pouvoir. Chacun a son idée de la façon selon laquelle la paroisse doit être gérée. Et pourquoi le prêtre aurait une idée meilleure que les laïcs? Personnellement, je trouve que ça s'oppose avec un rôle spécifique du prêtre qui n'est pas reconnu, le fait que c'est celui qui envoie en mission. On en revient toujours à des questions de conflits de pouvoir, de répartition des tâches ». Jeune prêtre du diocèse de Paris.

45. Dès 1969, Monseigneur Robé fonde à Bayeux un séminaire remettant au premier plan la formation spirituelle et cultuelle. Cette initiative ne reste pas isolée. Un an après, à Paray-leMonial, est constitué autour du Père Ladame un groupe de candidats à la prêtrise souhaitant recevoir une formation différente de celle prodiguée dans les structures diocésaines. Quelques années plus tard, des séminaires conformes à cet objectif de resacerdotalisation du clergé sont créés à Paris par Monseigneur Lustiger, à Ars par Monseigneur Bagnard, ainsi qu'à Toulon par Monseigneur Rey. Ils ont pour objectif de former des prêtres pieux et conscients de la spécificité irréductible que leur confère l'ordination.

46. Sous sa forme de confession auriculaire individuelle. 
On trouve indéniablement ici un rejet des modes d'autorité, faisant une large place aux laïcs, qui s'étaient développés notamment au sein de l'Action catholique ou encore dans le mouvement catéchétique. Ces mouvements avaient en effet pu constituer pour les laïcs des « relais aux revendications de participation aux décisions et à l'élaboration des lois et orientations diocésaines " ${ }^{47}$, contribuant ainsi à une transformation des rapports sociaux au sein de l'Église.

Pour finir, remarquons que Rome participe pleinement à ce mouvement de retour à un fort conformisme normatif en ce qui concerne la figure du prêtre. Ainsi un certain nombre de documents récents réaffirment-ils clairement l'attachement romain au modèle sacerdotal ${ }^{48}$, le terme de sacerdoce étant d'ailleurs désormais préféré à celui de presbytérat ${ }^{49}$. Le caractère irremplaçable du prêtre, ainsi que la distinction entre sacerdoce commun des baptisés et sacerdoce des ordonnés, s'y trouvent plusieurs fois réaffirmés. La configuration du prêtre au Christ est, elle aussi, rappelée en insistant sur le don de soi (à travers l'exemple du célibat) et sur la délivrance des sacrements. D'un point de vue disciplinaire, le port de l'habit ecclésiastique est présenté comme nécessaire. Enfin, des appels récurrents sont lancés à la sainteté du prêtre. Dans un contexte d'approfondissement de la division du travail religieux entre clercs et laïcs, cette réponse romaine vise à soutenir les prêtres dont le statut apparaît de plus en plus précaire : "Le retour actuel à la doctrine de Trente semble être motivé par une inquiétude, qui devient dans l'Église la source d'une fixation angoissée. Devant le rôle croissant des laïcs dans leur participation aux tâches pastorales, on veut redonner confiance aux prêtres en leur rappelant ce qu'ils sont seuls à pouvoir faire. On soupçonne une confusion entre le sacerdoce ministériel et le sacerdoce des fidèles " ${ }^{50}$, écrit le théologien Bernard Sesbouë.

\section{Une pleine inscription dans la modernité cependant}

Henri Bourgeois, dans un article qu'il consacre à ce qu'il appelle le "néoclassicisme catholique », dont la recomposition de l'idéal sacerdotal par les

47. Jacques PALARD, "L'institution catholique en recherches. L'acteur, le théologien et le sociologue " in Jacques PALARD, dir., Le gouvernement de l'Église catholique. Synodes et exercice du pouvoir, Paris, Cerf, 1997, p. 38.

48. On peut se référer notamment à l'« Instruction sur quelques questions concernant la collaboration des fidèles laïques au ministère du prêtre » (Actes du Saint-Siège, La Documentation Catholique, n 2171, décembre 1997, p. 1009-1018), ainsi qu'à différentes productions de la Congrégation pour le Clergé, notamment «Le prêtre, ministre des sacrements et guide de la communauté en vue du troisième millénaire " (La Documentation Catholique, $\mathrm{n}^{\circ} 2219$, oct. 1999, p. 883-902), et l'instruction datant du vendredi 18 octobre 2002 portant sur l'office du curé " pasteur et guide de la communauté paroissiale».

49. Ce qui constitue une différence notable par rapport à la période du Concile de Vatican II comme l'explique Maurice VIDAL in Joseph Doré, dir. et Maurice VIDAL, dir., Des ministres pour l'Église, Paris, Centurion-Cerf-Fleurus-Mame, 2001, p. 205.

50. Bernard SESBOUÉ, op. cit., p. 95. 
jeunes prêtres relève pleinement, insiste sur la coexistence dans ce mouvement entre traits classiques (les termes de conservatisme et de traditionalisme sont soigneusement évités par l'auteur) et aspects propres à la modernité, tout particulièrement "l'accent mis sur la spiritualité, l'importance de la rencontre et de la convivialité, le besoin de signes identitaires, la familiarité avec les techniques de communications, l'internationalisme, parfois un certain humour ${ }^{51}$. En ce qui concerne les jeunes prêtres, leur inscription dans la modernité passe principalement par leur accession au hors-travail ainsi que par leur quête de l'ascèse, deux phénomènes qui relèvent des thématiques sociales très actuelles de l'accomplissement et de la réalisation de soi.

\section{L'accession au hors-travail}

Dans nos sociétés modernes, travail et hors-travail se trouvent étroitement liés. Le temps contraint permet le temps libéré. Ce dernier n'est pas seulement considéré comme un temps de récupération mais aussi, avec l'appartenance à la société de loisir, comme une occasion de plein épanouissement de la personne. La vocation sacerdotale semble se distinguer de toutes les autres activités occupationnelles par son caractère total qui fait de l'homme du sacré un serviteur permanent de Dieu et de son peuple. Cette spécificité est d'ailleurs très fréquemment mise en avant par les prêtres pour mettre en évidence l'inadéquation des modèles profanes du métier et de la profession pour décrire le sacerdoce.

Or, ces mêmes prêtres, tout particulièrement les plus jeunes, insistent aussi sur la nécessité qu'ils éprouvent à connaître de véritables moments de horstravail. Ils aspirent, comme la plupart de leurs contemporains, à une séparation plus stricte entre le temps professionnel et les autres moments de la vie. Tous les jeunes prêtres avec lesquels je me suis entretenue, ont affirmé avoir des loisirs : activités culturelles et sportives. J'ai rencontré des prêtres cyclistes ${ }^{52}$, motards, mélomanes et même un pilote de montgolfière. Un grand nombre dispose d'une journée de congés par semaine, le plus souvent le lundi. Tous prennent des vacances en été, voire aussi pendant l'année. Un jeune prêtre tout récemment ordonné dans le diocèse de Paris, très soucieux au cours de l'entretien de donner une image de lui pleinement conforme à l'idéal sacerdotal et à ses dimensions sacrificielles, a été le seul à affirmer «trouver son repos dans son ministère ». Il a cependant précisé ultérieurement qu'il n'envisageait pas de passer un été sans consacrer deux semaines à faire de l'alpinisme. Dans un article au titre évocateur,

51. Henri Bourgeois, "Le néo-classicisme catholique ", Études, tome 394, n² 2, février 2001, p. 231.

52. La dernière édition du Championnat de France cycliste du clergé (manifestation sportive créée en 2000, ouverte aux prêtres, diacres et religieux) a eu lieu le $1^{\text {er }}$ mai 2004 dans la Drôme à Saint-Rambert-d'Albon. Le vicaire général du diocèse, récemment nommé, lui-même passionné de cyclisme, s'est particulièrement investi dans l'organisation de l'opération. 
"Mon curé à la plage » ${ }^{53}$, l'hebdomadaire $\mathrm{La}$ Vie rendait compte de témoignages de prêtres qui refusent désormais de consacrer leur temps libre de l'été aux camps scouts et autres groupes de jeunes, et qui souhaitent bénéficier de véritables vacances «pour soi ». Certains évoquaient les stratégies permettant de préserver leur tranquillité : ne pas prendre son téléphone portable, ne pas révéler sa destination. L'exemple même de Jean-Paul II se trouve mobilisé pour légitimer un rapport désormais décomplexé au hors-travail : «(...) alors que ses prédécesseurs allaient à Castelgandolfo qui est un Vatican bis - un palais avec les audiences, etc. -, lui va à la montagne. On dit même que, dans les débuts de son pontificat, quand il était au mieux de sa forme, il a envisagé de faire aménager une piscine à Castelgandolfo $(. ..) »^{54}$.

Les loisirs ont aussi un coût non négligeable. Si le site internet de la Mutuelle Saint-Martin propose à ses adhérents prêtres et religieux des séjours à tarifs préférentiels au cours des mois de basse saison dans des maisons de vacances et des pensions de famille, un grand nombre de prêtres séjournent chez leurs proches ou acceptent de prendre en charge un presbytère dans une région touristique (en échange de la gratuité de l'hébergement, ils rendent quelques services à la paroisse). La faiblesse de leur revenu ainsi que leur statut de célibataire limitent donc leur accès aux vacances.

Cette aspiration à des moments de hors-travail passe largement par la possibilité de disposer d'un véritable espace privé. L'accession à un logement en dehors du patrimoine immobilier de l'Église représente un coût important pour les prêtres qui doivent alors faire face à un ensemble de frais directs et indirects qu'ils n'assument pas en logeant dans un presbytère. Il demeure souvent le luxe de ceux, peu nombreux, qui gagnent un salaire en dehors du strict circuit ecclésial, prêtres au travail ou aumôniers d'établissements publics rémunérés par l'État. Le revenu mensuel moyen des prêtres diocésains est compris entre 730 et 1100 euros, l'ensemble regroupant la somme versée par le diocèse, les honoraires de messe et les avantages en nature (tout particulièrement le logement, le chauffage et le téléphone) $)^{55}$. Même si un grand nombre habite dans un presbytère ${ }^{56}$, ils entendent poser des limites nettes entre l'espace paroissial et celui de leur domicile.

«J'essaye de marquer franchement les limites. Ça, c'est un peu une spécificité des jeunes prêtres. Les anciens ont plus de mal avec ça entre la frontière public/privé. Moi, je sépare bien. J'habite au troisième étage et personne ne monte sans invitation. Làdessus je suis un peu intransigeant car sinon on est envahi très vite. Ici, c'est mon

53. Sophie CHARLIER, « Mon curé à la plage ", La Vie, nº 3022, semaine du 31 juillet 2003, p. 42-45.

54. Patrice Gourrier, op. cit., p. 110-111.

55. François MayauX, «L'Église et l'argent. La perception des Français, entre mythes et réalités ", Études, déc. 2003, no 3996, p. 47.

56. Les presbytères ont pris depuis plusieurs années la forme de "maisons paroissiales » où ont lieu quotidiennement permanences et réunions qui peuvent durer tard le soir. 
bureau et je travaille là. Les gens eux ont une vie privée et ils ne se doutent pas que nous aussi de temps en temps on a envie de voir nos amis et de ne pas être dérangé ». Jeune prêtre du diocèse de Valence.

Le fait de jouir d'une véritable vie privée est justifié en termes d'équilibre personnel. Mésestimer cette aspiration peut même apparaître comme dangereux. On peut à ce propos citer Julien Potel qui écrivait déjà à la fin des années 1970 : «Prendre du temps pour la culture sous toutes ses formes, pour les loisirs, pour entretenir des liens d'amitié, n'est pas un luxe pour une personne. (...) Trop de prêtres ont tendance à les oublier ou hésitent à consacrer un temps suffisant par crainte de ne pas être disponibles. Une saine hygiène de vie prévient une usure qui risque d'être source de désenchantement ${ }^{57}$. Un prêtre me parlait à ce propos de la nécessité "d'une fidélité à l'humain ". L'attention à l'humanité du prêtre s'est faite plus vive depuis quelques décennies, signe de l'atteinte portée à la conception du prêtre comme homme séparé. Elle se trouve combinée à l'idéal du don de soi, puisque comme l'affirme un prêtre d'une trentaine d'années interrogé dans l'article de La Vie: "Pour mieux donner du temps aux autres, il faut en prendre pour soi ».

Profondément moderne, ce refus de sacrifier son intimité et son confort est en rupture flagrante avec la figure du bon prêtre du XIX siècle, immolé, dépossédé, comme celle du curé d'Ars qui pour confesser les fidèles qui venaient vers lui en masse, allait selon la légende jusqu'à renoncer à ses heures de sommeil ${ }^{58}$. Cette aspiration a été prise en compte par la hiérarchie catholique, comme le montre l'article 283 du Code de droit canonique de 1983, qui dispose que les prêtres "bénéficieront (...) tous les ans d'une période de vacances convenable et suffisante ", rejoignant ainsi certaines affirmations de Presbyterorum ordinis. Les fidèles semblent eux aussi avoir pris conscience de cette nécessité. Ainsi dans les Actes du Synode du Diocèse de Valence, un certain nombre de recommandations sont faites à ce sujet : "Le synode se préoccupe de la qualité de la vie des prêtres. Pour cela il rappelle l'importance des vacances. Ils insistent pour que les vicaires forains veillent à ce qu'elles soient prises effectivement (...) Le synode demande à ce que l'on veille à laisser des soirs de la semaine sans réunion. Il demande que les fidèles soient attentifs au repos hebdomadaire des prêtres ${ }^{59}$.

L'image de l'homme "corvéable à merci » semble donc avoir fait long feu. L'abnégation trouve l'une de ses limites dans l'hédonisme de la société de loisirs. Les mortifications de l'abbé Donissan de Sous le soleil de Satan de Bernanos appartiennent bel et bien à un âge révolu. On retrouve ici un phénomène qu'ont

57. Julien Potel, Demain d'autres prêtres? Leur place et leur rôle, Paris, Le Centurion, 1977, p. 99.

58. Ce phénomène relève aussi de la thématique très moderne du risque de surmenage auquel les prêtres (comme de nombreux autres professionnels) participent pleinement et qui au $\mathrm{XX}^{\mathrm{e}}$ siècle a succédé à celui de l'oisiveté. Voir Christian PONSON, "La figure du prêtre dans l'histoire récente de l'Église : constantes et évolutions ", Prêtres diocésains, mars-avril 1998, pp. 147-166.

59. Actes du Synode, numéro spécial, Église de Valence, 1994, p. 68. 
aussi connu les pasteurs protestants, phénomène bien décrit par Jean-Paul Willaime ${ }^{60}$. Ces derniers ne veulent plus être considérés comme des «serviteurs souffrants » mais comme des spécialistes qui ont droit, eux aussi, à une réalisation de soi en dehors de leur travail, marquant ainsi le passage d'un " pastorat total » à la « profession de pasteur ${ }^{61}$.

\section{Performance de l'ascèse et réalisation de soi}

Le rapport à la contrainte dans ses dimensions sacrificielles, qui s'inscrit actuellement dans le registre de la performance de l'ascèse, vise une réalisation et non plus une abolition de soi. «N'est-ce (...) la plénitude de leur humanité qui se trouve sacrifiée ? Non. On s'imagine volontiers que l'héroïsme consiste à risquer sa vie (...) Mais ce n'est pas du tout ce que Jésus enseigne ! L'amour tel qu'il en révèle la profondeur et l'efficacité ne réside pas dans la destruction de soi (...) Se donner n'est pas se réduire à néant mais vivre ${ }^{62}$. Ces propos du Cardinal Lustiger reflètent très clairement ce phénomène. On est donc loin aujourd'hui de l'idéal du prêtre du XVIII et de celui du XIX ${ }^{e}$ siècle décrit par Dominique Julia comme « une sorte de neutre qui se définit par la catégorie du ni ni ; gommant toutes les aspérités de sa personnalité, (devant) s'effacer comme individu : l'élimination de tout trait propre, jointe à la séparation du monde, (étant) aux yeux des éducateurs du clergé moderne la condition de visibilité du sacerdoce christique $"{ }^{63}$. On peut, pour illustrer la question du rapport à la contrainte, reprendre l'exemple du célibat. Alors que dans la seconde moitié du $\mathrm{XIX}^{\mathrm{e}}$ siècle, il était perçu comme "un sacrifice, qui doit assurer tout à la fois l'autorité temporelle et le salut éternel » ${ }^{64}$, il est présenté dans le discours des jeunes prêtres que nous rencontrons comme "libérateur". Selon eux, ce n'est pas la religion et ses exigences qui sont aliénantes mais au contraire les quêtes sociales actuelles effrénées de jouissances physiques et matérielles, présentées comme des entraves à la véritable liberté.

Au sein de la modernité, la fascination pour la figure de l'aventurier ${ }^{65}$ avec laquelle celle du prêtre partage une indéniable proximité, provient du fait qu'il est perçu comme un "extrémiste de soi » ${ }^{66}$. L'affirmation et l'expression de soi

60. Jean-Paul Willaime, Profession: pasteur. Sociologie de la condition de clerc à la fin $d u \mathrm{XX}^{e}$ siècle, Genève, Labor et Fides, 1986.

61. Voir la préface de Roland CAMPICHE à l'ouvrage de Jean-Paul Willaime, op. cit.

62. Jean-Marie Lustiger, op. cit., p. 108.

63. Dominique JULIA, "L'éducation des ecclésiastiques en France aux XVII et XVIII ${ }^{\mathrm{e}}$ siècle ", Problèmes d'histoire de l'éducation. Actes des séminaires organisés par l'École française de Rome et l'Università di Roma-La Sapienza (janvier-mai 1985), Rome, École française de Rome, (collection de l'École française de Rome), nº 104, 1988, p. 219-220 et 228.

64. Philippe Boutry, op. cit., p. 236.

65. C'est la même métaphore à laquelle a recours Frédéric Mounier dans un article consacré à «L'image du prêtre pour les Français », La Croix, 22 juin 1996 : «Tous pour lui, lui pour tous. Être prêtre aujourd'hui : une aventure qui en vaut bien d'autres ».

66. Sylvain Venayre, La Gloire de l'aventure. Genèse d'une mystique moderne, 18501940, Paris, Aubier, 2002 (cité par DE SINGLY, op. cit., p. 66). 
nécessitent, comme le montre bien François de Singly, une "réalisation dans l'action " ${ }^{67}$ qui peut fonctionner sur le modèle de l'héroïsme auquel la performance de l'ascèse rattache les prêtres.

Si les jeunes prêtres se distinguent fortement du reste de leur classe d'âge, ils sont en phase avec un certain nombre de jeunes catholiques chez qui l'on trouve cette même volonté affichée du respect scrupuleux de la norme romaine et cette quête de l'ascèse. Le discours sur la morale sexuelle offre une bonne illustration de ce phénomène. L'audience dont bénéficie dans ces milieux un prêtre comme le père Daniel Ange, dont le principal message réside dans la défense de la virginité pré-conjugale, ou du professeur Joyeux, diffuseur des méthodes de contraception dites naturelles, est pour le moins stupéfiante. Le fait que coexiste au sein des communautés charismatiques (qui sont des communautés " jeunes") un attrait pour des formes liturgiques innovantes et un attachement farouche aux principes les plus traditionnels concernant les mœurs, participe du même mouvement. Au sein de certaines communautés nouvelles, le retour à des formes de piété traditionnelle telles que les adorations eucharistiques qui impliquent le corps, voir sollicitent la résistance physique lorsqu'elles se poursuivent tout au long de la nuit ${ }^{68}$, peut prendre une dimension ascétique. Les "goums ", sorte de retraites d'une semaine dans une région désertique créées par Michel Menu en 1969, connaissent un succès croissant auprès de cette même frange de croyants. Ils se font dans des conditions particulièrement spartiates : levée à l'aube, port d'un sac à dos rempli des provisions pour la semaine qui d'ailleurs se limitent en ce qui concerne la nourriture à du riz, marche d'une vingtaine de kilomètres par jour, nuit à la belle étoile... Cette version catholique des "raids aventures » qui connaissent un grand succès dans les directions des ressources humaines de certaines entreprises, a pour objet d' " approcher Dieu et se trouver soi-même ", "d'établir de vraies relations avec les compagnons ». Elle semble répondre aux quêtes très modernes d' "authenticité ", de " liberté » et de «fraternité " ${ }^{69}$ des participants.

L'invitation à l'ascèse, pratiquée par les clercs eux-mêmes ${ }^{70}$ et pouvant être reconnue socialement comme performance permettant une réalisation de soi

67. François de SINGLY, op. cit., p. 120.

68. Ainsi le groupe des Semeurs d'Espérance organise-t-il mensuellement une veillée de prière suivie d'une nuit d'adoration en l'église Saint-Séverin dans le $\mathrm{V}^{\mathrm{e}}$ arrondissement de Paris. Les participants invités à apporter leur sac de couchage se relaient, par petits groupes, jusqu'aux laudes à 7 heures du matin. Autre initiative plus ponctuelle, le Foyer de Charité de Châteauneufde-Galaure proposait à l'occasion du passage à la nouvelle année 2005, une nuit d'adoration s'étalant de 22 heures au lendemain 4 heures 45, moment de célébration d'une messe. Aïn Karem et l'Emmanuel en donnent d'autres exemples.

69. Ces différentes citations proviennent du site internet: www.goums.org

70. Ce qui rend leur discours vraiment crédible. Ascèse des clercs et ascèse des fidèles se trouvent étroitement liées. C'est un phénomène qu'a illustré Claude Langlois, en s'appuyant sur le XIX siècle, dans son intervention au colloque de l'AFSR de février 2002, intitulée « Le crime d'Onan ou la sexualité dans le débat public catholique ». L'historien a présenté l'usage contrôlé de la sexualité par les fidèles mariés et le célibat des clercs, comme répondant à la "nécessité de "partager" le modèle ascétique qui sous-tend l'architecture catholique ». 
pleine et heureuse, peut dès lors s'inscrire dans une démarche d'évangélisation, d'appel à la conversion. C'est ainsi que se dessine depuis quelques années une nouvelle pastorale, aujourd'hui encore controversée (prenant notamment la forme d'un conflit de générations) comme l'explique ce prêtre :

«On est dans deux logiques. Soit une logique un peu minimaliste selon laquelle il ne faut pas en demander trop, toute une pastorale de ces trente dernières années, une espèce de pastorale de l'accueil où l'on misait sur le look... en baissant de plus en plus les exigences... Au niveau des sacrements, de la catéchèse etc. Avec cette idée que l'important, c'est que les gens fassent un pas. Après avoir fait un pas, l'idée est qu'ils en feront un autre. C'est un raisonnement valable en période de chrétienté : l'important c'est que les gens bougent, après la société qui porte les valeurs chrétiennes les soutiendra. Maintenant, on n'a plus ce soutien. Aujourd'hui, ça s'effondre. Cette pastorale-là montre ses limites de façon de plus en plus évidente. L'autre attitude qui a eu du mal à se dessiner mais qui se dessine dans les communautés nouvelles, qui est de proposer les exigences de la foi chrétienne avec une vraie démarche d'évangélisation. Non pas simplement donner la loi, un certain légalisme. Mais de montrer combien les exigences du Christ sont libérantes et répondent aux attentes du cœur de l'homme. Cette pastorale est quand même délicate. Elle n'est valable que s'il y a vraiment un élan missionnaire. Ce ne sont pas des exigences qu'on plaque sur une demande religieuse, ce sont des exigences vers lesquelles on veut amener les gens, sachant qu'il faut les prendre là où ils sont. Il faut aller chercher les gens, il ne s'agit pas seulement de proposer. C'est dans ce sens-là que le texte des évêques de proposer la foi à la société française qui est remarquable par ailleurs, est encore en deçà. Il faut proposer la conversion. Toute la difficulté est de réaliser que les chrétiens ne sont plus chrétiens. (...) Ce qui est souvent mal compris, c'est l'exigence de conversion dont nous voulons être porteurs qui est perçue comme un intégrisme, une rigidité, un légalisme, vouloir que les gens rentrent dans un cadre précis sans faire attention à eux. Une conception assez juridique (...) Être porteur de cette exigence, ça me semble être le seul bras de levier, c'est un appel vers le haut. Refuser de brader les sacrements, c'est les rendre plus attirants. C'est ne pas les banaliser. Ça n'est pas une rigidité, un intégrisme, s'il y a vraiment une intention évangélisatrice. Il faut que j'amène les personnes qui sont devant moi à le réaliser et à le comprendre comme une bonne nouvelle pour eux. C'est ça souvent qui n'est pas vu. On nous dit : vous balayez toute une pastorale pour y mettre vos certitudes et votre désir identitaire etc., vous n'avez pas le respect des personnes. Or, on n'est pas du tout dans cette logique juridique. Le grand enjeu, c'est de se rendre compte que ces exigences peuvent être évangélisatrices ». Prêtre du diocèse de Paris, 39 ans, ordonné il y a 5 ans.

On perçoit à travers ce témoignage en quoi les recompositions du religieux propres à la haute modernité peuvent prendre tout autant une forme douce et distanciée que, dans un contexte de profonde incertitude, dure et fondamentaliste, ce qui n'empêche pas comme le rappelle Jean-Paul Willaime « de multiples interférences où l'on voit le religieux soft et rationnel s'émotionnaliser et s'intransigeanter et le religieux intransigeant et émotionnel se libéraliser et se rationaliser ${ }^{71}$.

71. Jean-Paul Willaime, Sociologie des religions, Paris, PUF « Que sais-je ? », 2004, p. 215. 


\section{Conclusion}

L'une des évolutions les plus marquantes concernant le ministère du prêtre au cours des quarante dernières années, réside indéniablement dans la banalisation de son statut. Le caractère total de son engagement faisait que le prêtre se trouvait au service de l'Église vingt-quatre heures sur vingt-quatre, toute sa vie, sans retraite possible, sans territoire à soi, très fortement dépendant de l'institution. La formation reçue, reposant essentiellement sur la philosophie et la théologie, faisait (et fait encore) de lui un généraliste mal à l'aise avec les disciplines plus appliquées que sont par exemple les sciences de l'éducation et la gestion. Ce modèle du sacerdoce total est entré en profonde contradiction avec un certain nombre d'évolutions historiques : contraintes démographiques conduisant à une pénurie de clercs, ébranlement de la civilisation paroissiale et de son ancrage territorial, progrès de la division du travail, mobilité accrue, mais aussi nouveau rapport à la temporalité. Tous ces phénomènes ont contribué à modifier en profondeur le mode d'exercice de la prêtrise en le rapprochant grandement des modèles professionnels profanes. C'est ce qui a été montré avec la question du rapport au hors-travail.

L'attachement si fort des jeunes prêtres diocésains à la contrainte, voire à l'ascèse, qui pouvait paraître pour le moins paradoxal, prend alors du sens. Il peut être analysé comme l'un des derniers moyens de renouer avec la figure remarquable et noble du virtuose spirituel dont le moine est l'expression idéale, avec une totalisation devenue aujourd'hui comme impossible. De plus, loin d'être un pur archaïsme, cette quête exprimée par des individus qui ont été immédiatement plongés dans un tel contexte, participe des thématiques pleinement modernes que sont la performance et la réalisation de soi. L'idéal sacerdotal se trouve donc ici davantage recomposé que réactivé, les jeunes prêtres offrant une bonne illustration du mouvement de "subjectivation du rapport à la religion ${ }^{72}$ décrit par Danièle Hervieu-Léger comme l'une des caractéristiques essentielles des croyants en haute modernité.

Céline BÉRAUD

Centre d'Études Interdisciplinaires des Faits Religieux

72. Danièle Hervieu-Léger, Catholicisme, la fin d'un monde, Paris, Bayard, 2003, p. 82. 
Mots-clés : clergé, vocation, idéal sacerdotal, performance, autorité, accomplissement personnel

\section{Résumé}

Les prêtres de la génération Jean-Paul II présentent des caractéristiques fort différentes de celles de leurs aînés. Une grande partie d'entre eux cultivent un style néo-tridentin (attachement à un mode de vie singulier et exigeant, soin porté à la liturgie, défense des prérogatives sacerdotales). La radicalité de leur choix de vie les inscrit dans le registre de la performance de l'ascèse. Cette virtuosité se trouve mise au service de leur accomplissement personnel, tout comme leur participation à la société de loisirs. Loin d'être un archaïsme, cette recomposition de l'idéal sacerdotal par les jeunes prêtres manifeste leur pleine intégration à la modernité.

\section{Abstract}

The priests of the generation John-Paul II show characteristics extremely different from those of their elder. A great part of them cultivate a neo-tridentin style (fondness for a singular and demanding way of life, care carried to the liturgy, defence of the sacerdotal prerogatives). The radicality of their choice of existence puts them in the register of the performance of the asceticism. This virtuosity participates in their personal achievement, just like their practice of leisure activities. Far from being an archaism, this recombining of the sacerdotal ideal by the young priests expresses their full integration into modernity.

\section{Resumen}

Los sacerdotes de la generación de Juan Pablo II presentan características muy diferentes de las de sus mayores. Una gran parte de ellos cultiva un estilo neo-tridentino (apego a un modo de vida singular y exigente, cuidado extremo de la liturgia, defensa de las prerrogativas sacerdotales). La radicalidad de su opción de vida los inscribe en el registro de la performance de la ascesis. Este virtuosismo se pone al servicio de su realización personal, así como su participación en la sociedad del ocio. Lejos de ser un arcaísmo, esta recomposición del ideal sacerdotal por parte de los jóvenes sacerdotes manifiesta su plena integración a la modernidad. 